\title{
Design for Dependability and Autonomy of a Wearable Cardiac and Coronary Monitor
}

\author{
José Machado da Silva, Cristina Oliveira, Bruno Mendes, Rúben Dias, Tiago Marques \\ INESC TEC and Faculty of Engineering, University of Porto \\ Porto, Portugal \\ Email: jms@fe.up.pt, cristina.oliveira@fe.up.pt, rtdias@inescporto.pt
}

\begin{abstract}
Wearable vital signals monitoring systems are promoting new assistance approaches in the healthcare system and are essential for the future connected health paradigm. The present work addresses the design of a cardiac and coronary monitoring system taking into consideration dependability and autonomy issues. A fuzzy logic approach is used to determine, in case deviations in the captured electrocardiogram are detected, whether these are occurring in the patient or in the system. As for autonomy, the use of data compression versus extra data processing balance is analysed to find operating conditions for which compression is worth to be used.
\end{abstract}

\section{INTRODUCTION}

New, prevention based, healthcare paradigms can be accomplished after continuously monitoring vital signals with wearable systems. These provide information on the wearers' health status and allow for a faster and eventually more correct intervention of emergency medical technicians.

According to the European Council Directive 90/385/EEC, active implantable medical devices “... must be designed and manufactured in such a way that (...) their use does not compromise the clinical condition or the safety of patients. They must not present any risk to the persons implanting them or, where applicable, to other persons." [1].

The dependability of a system is determined by different attributes (e.g., availability, security and maintainability), risks (defects and failures), and means (of prevention, detection and fault tolerance) and its analysis enables for developing efficient testing and maintenance methodologies.

The first step in the development of a medical monitoring device is identifying the risks of not achieving acceptable levels of dependability. After this, fault detection and fault tolerance/reconfiguration techniques can be used to detect system malfunctions and correct them [2].

Autonomy also compromises dependability. Low-power is achieved after using power efficient devices and by means of adaptively managing power consumption during system operation. E. g., data sampling and transmission rates can be reduced in case the wearer is in a normal health condition, or different priorities can be dynamically assigned to capture and transmit vital signals according to patients' condition [3].

This paper presents the design for dependability and autonomy of a combined cardiac and coronary surveillance system (section II), aiming at developing a wearable cardiac monitor for post-EVAR (endovascular aneurysm repair) surveillance. The developments presented here regard the dependability

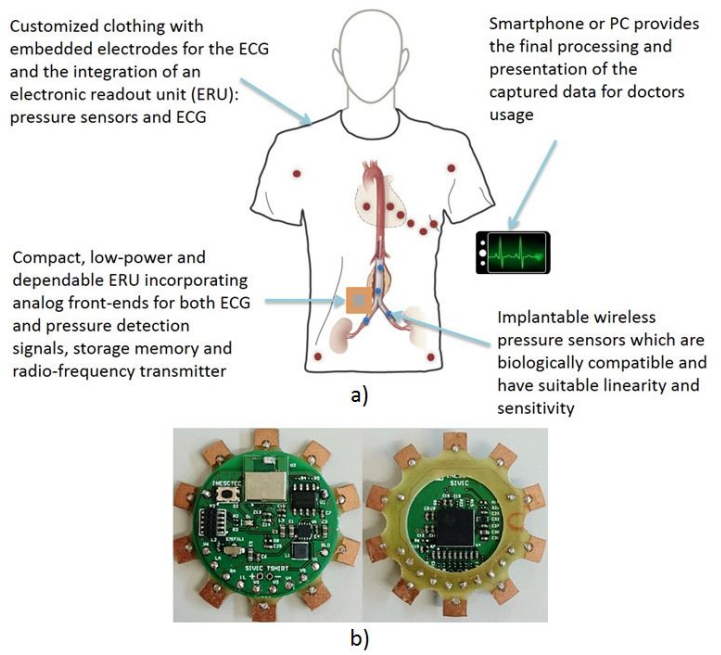

Fig. 1: a) SIVIC system b) The 12-lead ECG data acquisition and transmission module.

strategy (section III) relying on data fusion techniques and the use of data compression to reduce the volume of data to be transmitted and thus increase the system autonomy (section IV). The main conclusions are highlighted in section V.

\section{COMBINED CARDIAC AND CORONARY SURVEILLANCE SYSTEM}

The wearable combined cardiac and coronary surveillance (SIVIC) system (Figure 1) provides the synchronous capture of the ECG (electrocardiogram) and of the pressure in the abdominal aneurysm sac. Wireless pressure sensors are used to capture the intra-sac aneurysm pressure [4]. An electronic readout unit (ERU) capable of energizing the sensors and capture the pressure data is placed on the chest of the patient. Data is transmitted to a smartphone for further processing, data display, and eventual communication with a healthcare center.

Figure 1.b) shows the customized 12-lead ECG data acquisition and transmission (DAT) module prototype. It comprises an ECG acquisition analogue front-end (AFE) based on the low-power (0.75 mW/channel) TI 24-bit ADS1298 chip and a PAN1740 Bluetooth Low Energy (BLE) module based on the Dialog DA14580 SoC. This SoC includes a 32 bit ARM 
Cortex M0 microcontroller $(\mu \mathrm{C})$ running at $16 \mathrm{MHz}$, that is used to perform all the necessary processing operations. The BLE module presents an advertised power consumption of 4.9 $\mathrm{mA}$ when transmitting/receiving.

Wireless ECG monitoring systems with a high number of leads (e.g. 12-lead) are usually designed for clinical usage, being systems with a lower number of leads (e.g. 1 to 3 leads) commonly used in ambulatory cases [5]. Our system was designed having in mind its use in both scenarios and thus the number of ECG data acquisition channels is reconfigurable. Inputs not used to capture ECG signals can be used to acquire other biosignals.

The chosen $250 \mathrm{~Hz}$ ECG sampling frequency provides a good balance in terms of data accuracy and power consumption. While no significant difference is found between ECGs sampled at $500 \mathrm{~Hz}$ and $250 \mathrm{~Hz}$, that is not the case with the difference between ECG traces sampled at $250 \mathrm{~Hz}$ and 125 $\mathrm{Hz}$ as a significant difference can be detected in terms of peak amplitude reduction and interval measurement.

The system has three main states of operation: initialization, wait for connection, and running. In the running mode, the $\mu \mathrm{C}$ receives an interruption from the ADS1298 every $4 \mathrm{~ms}$, indicating the presence of new samples. That initiates an SPI communication to get the samples from the $\mathrm{N}(\leq 8)$ captured channels and store them in a buffer. Each $\mathrm{T}$ ms, where $\mathrm{T}$ can be defined, an application task is executed to check if the buffer size reached 120 bytes. When that is the case, data is copied to the BLE Attribute Protocol (ATT) database and a notification is sent to the mobile phone to initialize the data transmission procedure. The final ECG data processing operations (e. g., decompression, QRS and T-wave detection algorithms) are implemented in the mobile phone.

It is commonly accepted that a wireless ECG capture system should ensure a data transmission of $4 \mathrm{kbps}$ and a maximum latency of $500 \mathrm{~ms}$ should be verified in the transmission of each data packet. This allows us to adapt $\mathrm{T}$ according to the most convenient interval.

Figure 2 shows (blue bars) the power supply current $\left(V_{D D}=3.3 \mathrm{~V}\right)$ of the DAT module in three modes of operation. The Running value corresponds to the case of the DAT performing its mission with a T interval of $10 \mathrm{~ms}$. An autonomy of about 1.5 days would be obtained with a 100 $\mathrm{mAh}$ battery. The orange bars give the current consumed when the three main tasks are performed isolated, i. e., the same operation is performed sequentially in a loop. Transmitting Data: continuously send data to the mobile phone - consumption dominated by the BLE module; Only Processing - the ADS1298 is turned off and the $\mu \mathrm{C}$ performs sum operations; Lower Power State- the ADS1298 is turned off and the $\mu \mathrm{C}$ is in sleep mode. It can be seen that, although a BLE link is used, data transmission is the operation with the highest power consumption - about 3.5 times more than processing.

\section{FUZZY LOGIC BASED DEPENDABILITY STRATEGY}

The morphologies (amplitude and interval/segment length) taken by the three main events of an ECG cycle, the P wave,

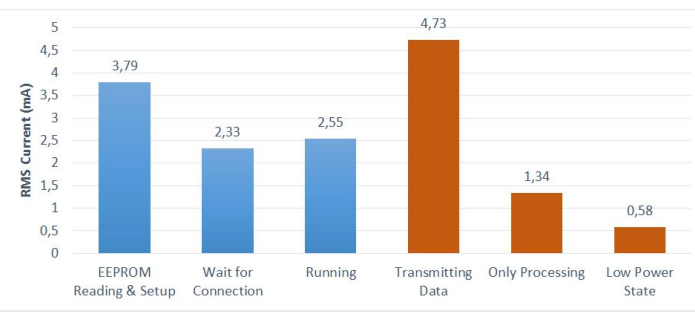

Fig. 2: Power consumption of each one of and main tasks.

the QRS complex, and the T wave vary according to a person's physiological condition.

Since wearable devices are more affected by noise, filtering of the ECG is a necessary pre-processing step to reduce noise components while preserving the QRS complex shape, in order the heart rate $(\mathrm{HR})$ - in beats per minute (bpm) - can be accurately measured. The Pan-Tompkins algorithm is used for ECG filtering and the HR calculation [6].

The availability of different sensors in wearable systems allows for fusing the respective data to formulate better decisions from the captured data. Other biosignals, such as the blood pressure (BP), defined by the systolic (maximum) and diastolic (minimum) pressures, can provide information on the patient condition, eventually affected by physical activity or diseases. Accelerometers enable tracking the wearer activity that might influence the heart activity. The SIVIC system also includes an electrode-skin impedance measuring circuit, which allows detecting if the electrodes are connected to the patient or are loose/disconnected.

Data fusion techniques have been applied as a means for combined analysis of several physiological signals that can potentially provide additional information on a patient's condition. Kenneth et. al performed the fusion of ECG, blood pressure, saturated oxygen content and respiratory data to achieve improved clinical diagnosis of patients in cardiac care units [7]. In our case, as a first approach, a fuzzy logic system is used to fuse data due to its probability assignment based on rules. Since the values of the features extracted from the biosignals can be assigned in regions well defined in the medical domain, defining rules is relatively straightforward (Table I).

Our approach also includes features that characterize the system functionality to estimate the Global Status. The signalto-noise ratio (SNR) of bioelectrical signals is known to be related to the electrode-skin impedance [8]. Since this impedance varies for each person and is affected by factors like temperature and applied pressure, the electrode-skin impedance is measured when the system is switched on and is monitored periodically to establish a normal region for the impedance values for which the acquired ECG quality is considered acceptable. These values are then used to assess the measured impedance during normal operation of the system. In case the impedance values are higher than expected, signalling a potential loose connected electrodes situation, the fuzzy logic system updates the System Status. 
TABLE I: Fusion rules for patient condition diagnosis.

\begin{tabular}{|l|l|l|}
\hline Signals & Condition & Rule \\
\hline \hline \multirow{3}{*}{ ECG } & Normal & HR between 60 and $100 \mathrm{bpm}$ \\
\cline { 2 - 3 } & Asystole & No QRS for at least 4 seconds \\
\cline { 2 - 3 } & Extreme Bradycardia & $\begin{array}{l}\text { HR lower than } 40 \mathrm{bpm} \text { for } 5 \\
\text { consecutive beats }\end{array}$ \\
\cline { 2 - 3 } & Extreme Tachycardia & $\begin{array}{l}\text { HR higher than } 140 \mathrm{bpm} \text { for } 17 \\
\text { consecutive beats }\end{array}$ \\
\hline \multirow{4}{*}{ BP } & Normal & $\begin{array}{l}\text { systolic: } 90-139 \mathrm{mmHg} \\
\text { diastolic: } 60-89 \mathrm{mmHg}\end{array}$ \\
\cline { 2 - 3 } & Hypotension & $\begin{array}{l}\text { systolic: }<90 \mathrm{mmHg} \\
\text { diastolic: }<60 \mathrm{mmHg}\end{array}$ \\
\cline { 2 - 3 } & Hypertension & $\begin{array}{l}\text { systolic: }>140 \mathrm{mmHg} \\
\text { diastolic: }>90 \mathrm{mmHg}\end{array}$ \\
\hline
\end{tabular}

A fuzzy logic system comprises 4 main components: fuzzy rules (knowledge base), fuzzy sets, fuzzy inference engine and defuzzification [9]. The inputs are the features previously extracted from the measured signals. The outputs are the quantities Patient Status, System Status and the Global Status. These provide a system diagnosis assessment from which a final decision is taken - i. e., either the patient has a health condition or the monitoring system is malfunctioning. The outputs are determined based on the input values of the fuzzy sets and the assigned rules for each output. The rules to define the Patient Status are based in medical data, the rules for the System Status are defined from the system specifications, and the rules for the Global Status include both. The fuzzy sets include the HR for each channel, the blood pressure (systolic and diastolic), the contact resistance, and can also include the acceleration if this data is available. Acceleration data increases the robustness of the fuzzy system, making it more flexible for an unobtrusive use.

A trapezoidal curve was chosen for the membership function. This is a function of a vector $\mathrm{x}$, and depends on four scalar parameters $a, b, c$, and $d$ (equation 1). The parameters $a$ and $d$ locate the "feet" of the trapezoid and the parameters $b$ and $c$ locate the "shoulders".

$$
\mu_{\text {trapezoidal }}(x)= \begin{cases}0, & x<a \text { or } x>d \\ \frac{x-a}{b-a}, & a \leq x \leq b \\ 1, & b \leq x \leq c \\ \frac{d-x}{d-c}, & c \leq x \leq d\end{cases}
$$

Table I shows the normal values for the HR and BP, and some examples of pathologies.

Data from the MIT Multiparameter database (MGH/MF) was used to test the fuzzy logic system using Matlab [10], [11]. The features from ECG signals (leads I, II and V) and the arterial blood pressure (ART) were extracted and feed to the fuzzy logic system. The ECG provides the HR information and the ART waveform is used to know the systolic and diastolic pressures. Also an impedance signal was added to the set in order to test for possible faults.

The fuzzy logic was evaluated for 3 situations:

1) One of the ECG channels (lead I) does not provide useful information, most likely due to a faulty contact. The other channels (leads II and V) enable the detection of the HR. The electrode-skin impedance value enables detecting the problem is related to the electrode. Result: Patient Status: 81; System Status: 18; Global Status: 81.

2) The recorded signals show SNR values good enough to identify relevant features, but the patient's blood pressure is very high (record MGH085 from the MGH/MF database). The System Status is ok, but the Patient Status indicates a health problem. Result: Patient Status: 14; System Status: 86; Global Status: 86.

3) Atrial flutter (arrhythmia) is recognized on an ECG by the presence of higher frequency waves superimposed to the normal beating (Figure 3). In this case the HR is calculated using lead V, and the ART waveform is also used for a more reliable HR estimation, since these signals are related. Result: Patient Status: 14; System Status: 86; Global Status: 86.

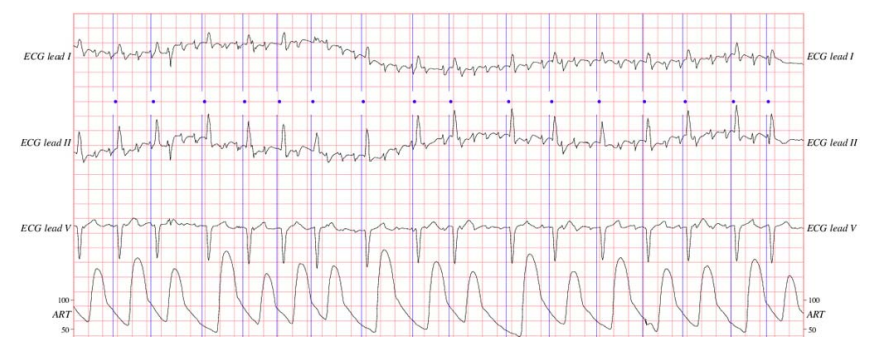

Fig. 3: MGH023 record: Atrial flutter. (Grid intervals: time $0.2 \mathrm{~s}$, ECG $0.5 \mathrm{mV}$, ART $25 \mathrm{mmHg}$ )

When the data fusion model detects that the System Status is degraded, built-in self tests can be performed to determine the cause. The smartphone sends an order for specific tests to be performed depending on the signals features. For instance, if an ECG channel presents a behaviour similar to the atrial flutter condition, but the remaining channels are normal, the origin of the flutter could be an oscillation in the ECG amplifier rather than the patient's heart. A simple test would be to interconnect both inputs of the amplifier and observe if the flutter persists. If not, in case the signal is really displaying a health condition that is then more visible in this particular ECG channel.

\section{DATA COMPRESSION FOR INCREASED AUTONOMY}

In data compression a common approach is to use a mix of lossy and lossless methods so that the best compression combination can be achieved. The performance of an ECG compression algorithm is commonly evaluated after two parameters [12]: the compression ratio (CR) - the ratio between the length of the output stream and the length of the original stream; and the percentage root-square difference (PRD) between the original signal and the reconstructed one (equation 2), which actually corresponds to a distortion measurement.

$$
P R D=\sqrt{\frac{\sum_{0}^{N-1}\left(x_{n}-\hat{x}_{n}\right)^{2}}{\sum_{0}^{N-1} x_{n}^{2}}} \times 100
$$


TABLE II: a) Compression and distortion levels obtained with three transforms. b) Compression levels after coding. (a)

\begin{tabular}{|l|c|c|}
\hline Transform & CR [\%] & PRD [\%] \\
\hline \hline Wavelet & 87.5 & 0.39 \\
\hline Cosine & 62.3 & 12.3 \\
\hline Fourier & 6.5 & 0.01 \\
\hline
\end{tabular}

(b)

\begin{tabular}{|l|c|}
\hline Algorithm & CR [\%] \\
\hline Ziv-Lempel & 81.3 \\
\hline Huffman & 75.7 \\
\hline RLE & 67.2 \\
\hline
\end{tabular}

In order to evaluate the best modelling approach to compress the captured ECG before transmission, three transforms were evaluated - Wavelets, Cosine, and Fourier. Table II(a) shows the CR and PRD values obtained after applying these transforms to the original ECG signal. It can be seen that the best relation between $\mathrm{CR}$ and $\mathrm{PRD}$ is achieved with the Wavelet transform.

As for the coder the dictionary-based Ziv-Lempel, Huffman, and the Run Length Encoding (RLE) methods were evaluated. In the three cases the input data was obtained after a 3level Haar Wavelet transform. The results in Table II(b) show that the highest compression would be obtained with the ZivLempel algorithm, but with this algorithm, as well as with Huffman's, besides the resulting compressed stream, it would be necessary to transmit also the constructed dictionaries so that encoded signals can be recovered at the receiving device. Also, when comparing the complexity of the codes and the time needed to process them, it was found that the Huffman and the RLE algorithms are processed $45.3 \%$ and $89.9 \%$, respectively, faster than for Ziv-Lempel. For this reason it is considered that the Wavelet-RLE approach is the best choice for our system. Introducing data compression each 256 bytes of data are compressed to 54 bytes. After reception in the smartphone the ECG is recovered with a PRD of $1.21 \%$.

Figure 4 shows that a reduction of about $7 \%$ in the power consumption is obtained after using compression. It is also shown that power consumption does not vary significantly with the time interval imposed between transmissions. As the global power consumption is dominated by the AFE of the data acquisition process, in this case the use of compression does not contribute significantly to save energy.

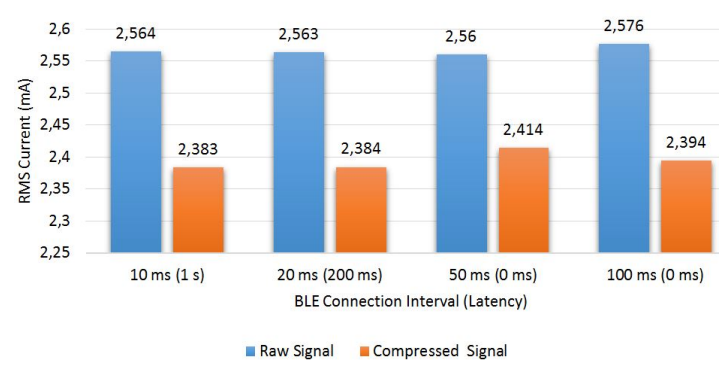

Fig. 4: Current consumed before and after data compression.

\section{CONCLUSION}

The present work shows how data fusion, notably fuzzy logic, can be explored to improve the dependability of a cardiac and coronary monitoring wearable system, after providing a means to diagnose whether deviations detected in the acquired signals are due to a disease or condition of the patient, or actually to a fault in the system. It is also a tool that can help the process of identifying test operations needed to improve the system's diagnosability.

Wearable systems are also required to present high autonomy levels. Although data compression has been proposed as an approach to reduce wireless communication transactions, it is shown that, in case continuous monitoring is required, energy savings obtained after data compression can be minimal comparing to the extra data processing and particularly the consumption imposed by the analogue front-end.

\section{ACKNOWLEDGMENT}

This work is financed by the ERDF - European Regional Development Fund through the COMPETE Program (operational program for competitiveness) and by National Funds through the FCT - Fundação para a Ciência e a Tecnologia (Portuguese Foundation for Science and Technology) within project SIVIC PTDC/EEI-ELC/1838/2012 (FCOMP-01-0124-FEDER028937) and grant contract SFRH/BD/81476/2011.

\section{REFERENCES}

[1] E. S. Organisations, Active Implantable Medical Devices. DirectorateGeneral for Internal Market, Industry, Entrepreneurship and SMEs Cosmetics and Medical Devices, 1990.

[2] F. L. Floch, S. Bernard, G. Bontorin, F. Soulier, , and G. Cathebras, "Global strategy to guaranty dependability of electrical medical implanted devices," in Proc. 5th International IEEE/EMBS Conference on Neural Engineering (NER), 2011, pp. 515-518.

[3] H. Kim, C. Van Hoof, and R. Yazicioglu, "A mixed signal ecg processing platform with an adaptive sampling adc for portable monitoring applications," in Engineering in Medicine and Biology Society, EMBC, 2011 Annual International Conference of the IEEE, Aug 2011, pp. 2196-2199.

[4] C. Oliveira, A. Sepúlveda, N. Almeida, B. Wardle, J. Machado da Silva, and L. Rocha, "Implantable flexible pressure measurement system based on inductive coupling," Biomedical Engineering, IEEE Transactions on, vol. 62, no. 2, pp. 680-687, Feb 2015.

[5] M. K. Delano and C. G. Sodini, "A long-term wearable electrocardiogram measurement system," in Body Sensor Networks (BSN), 2013 IEEE International Conference on, May 2013, pp. 1-6.

[6] J. Pan and W. J. Tompkins, "A Real-Time QRS Detection Algorithm," IEEE Transactions on Biomedical Engineering, vol. BME-32, no. 3, pp. 230-236, Mar. 1985.

[7] E. Kenneth, A. U. Rajendra, N. Kannathal, and C. M. Lim, "Data fusion of multimodal cardiovascular signals," in Engineering in Medicine and Biology Society, 2005. IEEE-EMBS 2005. 27th Annual International Conference of the, 2005, pp. 4689-4692.

[8] C. Oliveira, J. Machado da Silva, I. Trindade, and F. Martins, "Characterization of the electrode-skin impedance of textile electrodes," in Conf. on Design of Circuits and Integrated Systems (DCIS), Nov 2014.

[9] L. A. Zadeh, "Fuzzy Logic," Computer, vol. 21, no. 4, pp. 83-93, Apr. 1988.

[10] A. L. Goldberger, L. A. N. Amaral, L. Glass, J. M. Hausdorff, P. C. Ivanov, R. G. Mark, J. E. Mietus, G. B. Moody, C.-K. Peng, and H. E. Stanley, "PhysioBank, PhysioToolkit, and PhysioNet Components of a New Research Resource for Complex Physiologic Signals," Circulation, vol. 101, no. 23, pp. e215-e220, Jun. 2000.

[11] J. Welch, P. Ford, R. Teplick, and R. Rubsamen, "The Massachusetts General Hospital-Marquette foundation hemodynamic and electrocardiographic database - comprehensive collection of critical care waveforms," J Clinical Monitoring, vol. 7, no. 1, pp. 96-97, 1991.

[12] A. Agarwal, A. Sharma, and M. L. Devel, "ECG data compression for MIT-BIH record no. 100/ml II with DCT and DCT-2 frequency transformation techniques," MIT International Journal of Electrical and Instrumentation Engineering, vol. 3, no. 1, pp. 9-11, 2013. 